\title{
Accuracy of linear measurements made on cone beam computed tomography scans at different magnifications
}

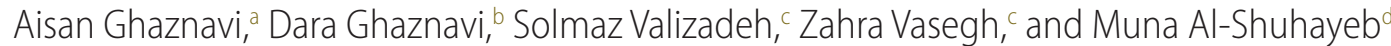

\author{
aDepartment of Oral and Maxillofacial Radiology, School of Dentistry, Urmia University of Medical Sciences, Urmia, Iran. \\ bDepartment of Periodontics, Faculty of Dentistry, Tabriz University of Medical Sciences, Tabriz, Iran. \\ cDepartment of Oral and Maxillofacial Radiology, School of Dentistry, Shahid Beheshti University of Medical Sciences, Tehran, Iran. \\ dDepartment of Periodontics, School of Dentistry, Shahid Beheshti University of Medical Sciences, Tehran, Iran. \\ Correspondence to Asian Ghaznavi (email: aisanghaznavi@yahoo.com). \\ (Submitted: 12 June 2019 - Revised version received: 28 July 2019 - Accepted: 04 August 2019 - Published online: 26 October 2019)
}

\begin{abstract}
Objective This study aimed to evaluate the accuracy of linear measurements made on cone beam computed tomography (CBCT) (New Tom VGi) scans with different image magnifications.

Methods Forty-two titanium pins were inserted into seven dry sheep mandibles. Lengths of the pins were measured using a digital caliper with $0.01 \mathrm{~mm}$ readability, and the mandibles were radiographed using a CBCT unit. After reconstruction of CBCT images, three experienced radiologists measured the length and diameter of titanium pins at 100\%,200\% and 400\% magnifications. Accuracy of measurements was analyzed using descriptive statistics and one-way analysis of variance. $P<0.05$ was considered statistically significant. Interobserver reliability was calculated using the intraclass correlation coefficient test.

Results The mean differences of linear measurements from the actual lengths of pins were $0.1960,0.2143$ and $0.2047 \mathrm{~mm}$ at $100 \%, 200 \%$ and 400\% magnifications, respectively $(P>0.05)$. The mean differences of linear measurements from the actual diameters of pins were $0.2206,0.2063$ and $0.1984 \mathrm{~mm}$ at $100 \%, 200 \%$ and $400 \%$ magnifications, respectively $(P>0.05)$. Interobserver reliability of pin length measurements was estimated to be $0.285,0.707$ and 0.479 at $100 \%, 200 \%$ and $400 \%$ magnifications, respectively. Interobserver reliability of pin diameter measurements was $0.078,0.469$ and 0.587 at 100\%, 200\% and $400 \%$ magnifications, respectively.

Conclusion Based on the results, image magnification does not affect the accuracy of linear measurements made on CBCT scans. Interobserver reliability of pin length measurements was good at 200\% magnification, while it was below the acceptable range at other magnifications. For measurement of pin diameters, this index was below the acceptable range at all magnifications.

Keywords cone beam computed tomography, image magnification, linear measurement
\end{abstract}

\section{Introduction}

Before the introduction of cross-sectional imaging, intraoral periapical and panoramic radiography were the most commonly used imaging techniques for implant treatment planning. These radiographic methods could only provide two-dimensional images of three-dimensional structures. Since 1980s, multidetector computed tomography (MDCT) has been the most accurate imaging technique for evaluation of implant site. However, MDCT has certain limitations such as high radiation dose, reduced image quality due to metal artifacts and high cost. Furthermore, non-isotropic voxels could lead to lower resolution of the reconstructed images compared with original axial scans. In the1990s, tomography systems such as cone beam CT (CBCT) were introduced for the head and neck imaging to overcome the limitations of CT. ${ }^{1,2}$

In the recent years, CBCT has been increasingly used in different fields of dentistry ${ }^{3,6}$ with several applications in implant treatment. ${ }^{7}$ One of the advantages of CBCT software for dentists is the possibility to make linear measurements between anatomical locations. Normally, linear measurements reused to determine the thickness and height of the alveolar ridge for the assessment of implant site before surgery, as well as to measure the distances between anatomical landmarks in orthodontics and calculate the size of pathological lesions of the jaws. Since linear measurements are required for implant therapy, orthodontic treatment and surgical management of pathological lesions, clinicians need to be aware of the accuracy of linear measurements made on CBCT scans, and the effects of different enhancement features on these measurements. ${ }^{8-9}$
Similar to other digital imaging software programs, CBCT offers various enhancement features to modify image quality based on the preferences of the user. Primary features used to enhance image quality are magnification (zoom, window/ level) and annotation. Moreover, cursor-driven algorithms enable accurate real time measurements. ${ }^{10,11}$

Several studies have evaluated the effects of parameters such as voxel size, thickness and reconstruction angle on the accuracy of CBCT linear measurements. The effects of magnification on the diagnostic accuracy of two-dimensional digital radiography have also been the subject of several studies. ${ }^{12-19}$ According to the literature; no study has assessed the accuracy of linear measurements made on CBCT scans with different image magnifications. Therefore, this study aimed to assess the effects of image magnification on the accuracy of linear measurements made by New Tom VGi CBCT device (New Tom VGi, Quantitative Radiology, Verona, Italy).

\section{Materials and Methods}

In this study, 42 titanium pins with equal dimensions were inserted into seven dry sheep mandibles. Before the placement of implants in the mandibles, pin lengths and diameters were measured using a digital caliper with $0.01 \mathrm{~mm}$ accuracy (Mitutoyo Corp., Kawasaki, Japan) (Figs. 1 and 2). Pin lengths were measured from the top to the bottom. Since pin diameters were not equal along the pin, square-shaped pin heads were considered as the main indicator of the diameter. 


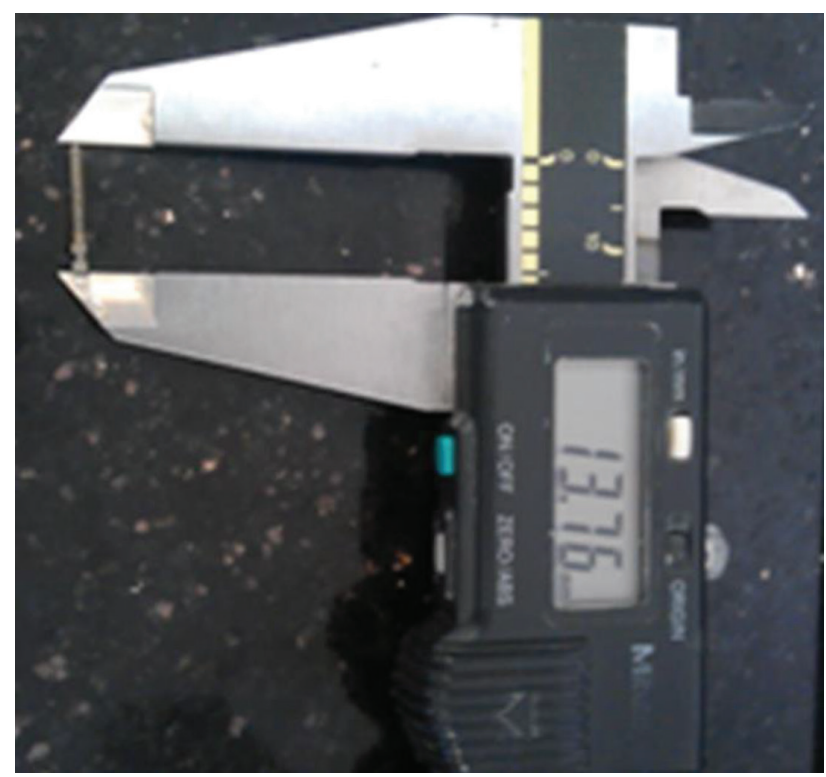

Fig. 1 Pin length measurement by digital caliper.

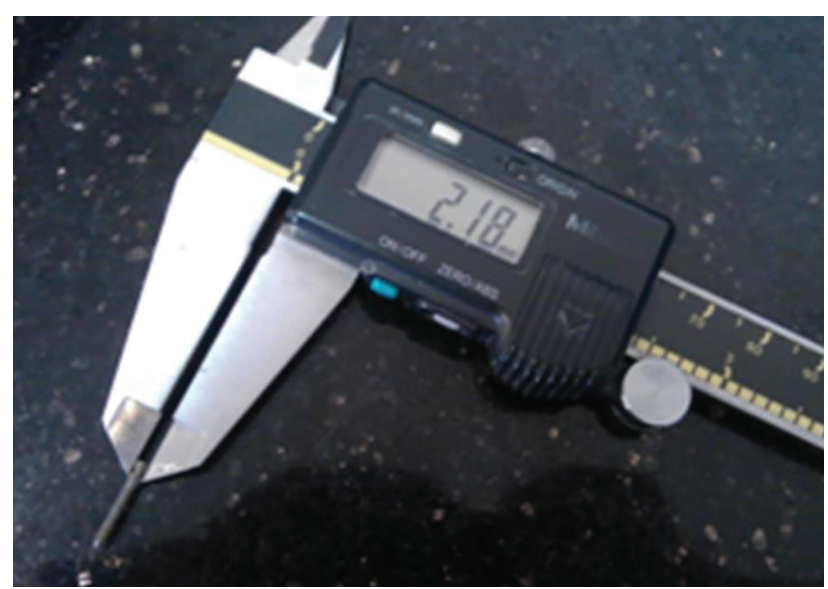

Fig. 2 Pin diameter measurement by digital caliper.

To facilitate measurement in the axial plane, diameter of the pin heads was considered as the diameter of the pins.

In order to insert the pins into the bone, first, parallel holes were drilled in the edentulous alveolar ridge crest. Afterward, the holes were widened to the diameters of the pins using high-speed hand piece and a diamond fissure bur, and the pins were finally inserted into the mandibles (Figs. 3 and 4).

Mandibles were placed in a container filled with water to simulate soft tissues. Afterwards, they were placed in the holder of the CBCT device (New Tom VGi, Quantitative Radiology, Verona, Italy). To standardize the position of the mandibles in the CBCT device, midsagittal and occlusal planes were adjusted to the vertical and horizontal laser markers, respectively. The mandibles were radiographed using $8 \times 12 \mathrm{~cm}$ field of view at $110 \mathrm{kVp}$.

After image reconstruction by NNT Viewer software, linear measurements were made by three oral and maxillofacial radiologists at $100 \%, 200 \%$ and $400 \%$ magnifications. The observers were allowed to change the contrast, sharpness and brightness of the images, which were displayed on a monitor with $1024 \times 1280$ pixels resolution and 32-bit color depth.

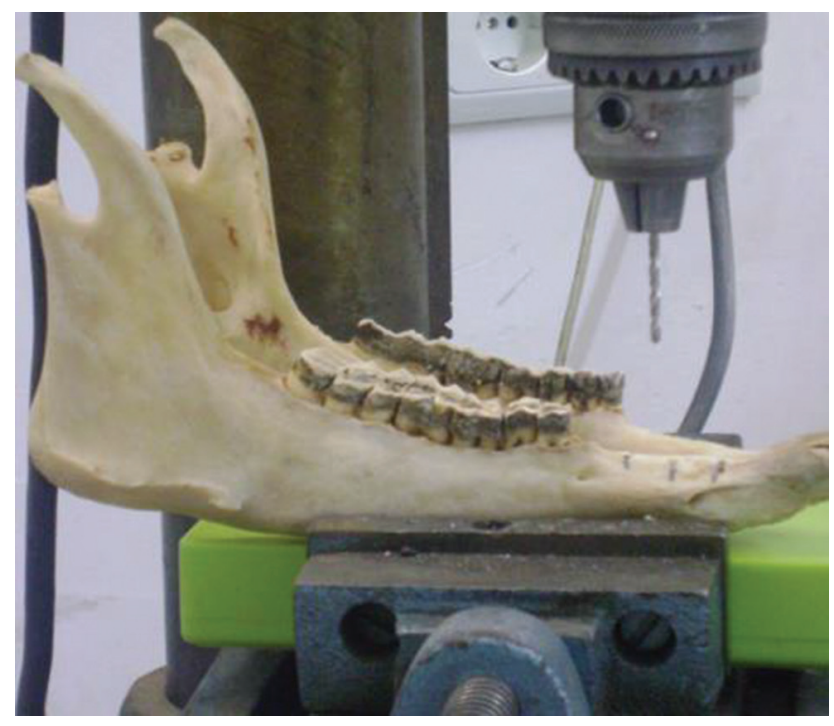

Fig. 3 Parallel drilling of implant holes.

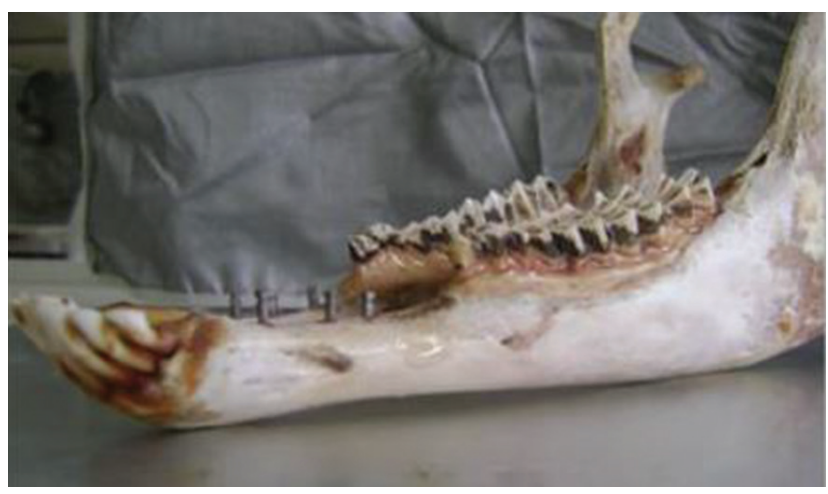

Fig. 4 Pins inserted into dry sheep mandibles.

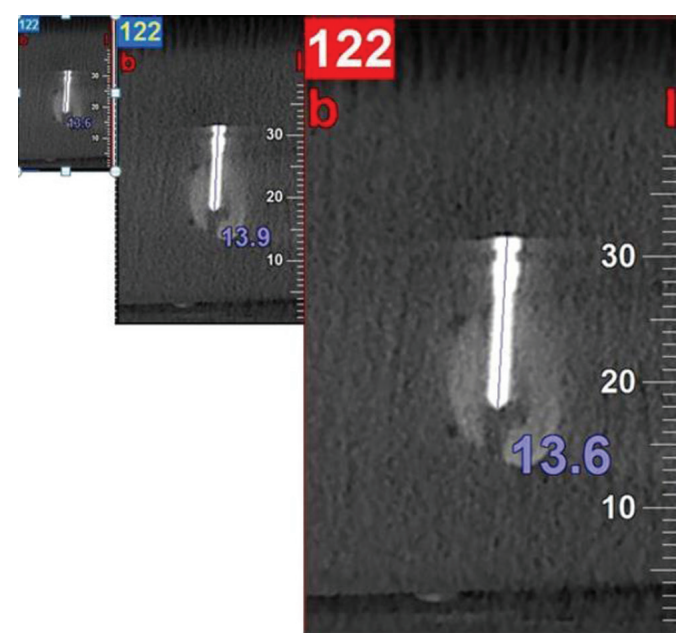

Fig. 5 Linear measurement of pin lengths at $100 \%, 200 \%$ and $400 \%$ magnifications.

To radiographically measure the lengths, overall length of the pins was measured in the cross-sectional plane. In addition, square heads of the pins were measured in the axial plane to estimate the pin diameters (Figs. 5 and 6).

Accuracy of linear measurements of the lengths and diameters of the pins in the axial and cross-sectional planes was 


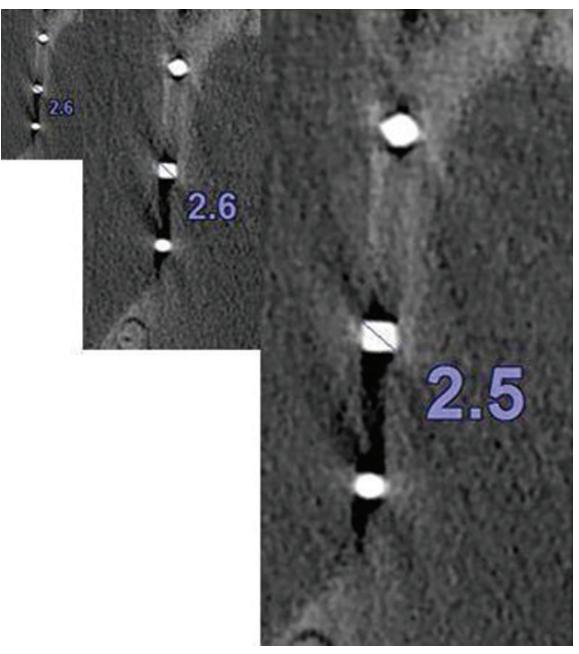

Fig. 6 Linear measurement of pin diameters at $100 \%, 200 \%$ and $\mathbf{4 0 0 \%}$ magnifications.

\begin{tabular}{lcccc}
\hline \multicolumn{5}{l}{$\begin{array}{l}\text { Table 1. Error ranges of pin length measurements at all } \\
\text { magnifications by the three observers }\end{array}$} \\
\hline Error range & $\begin{array}{c}\text { First } \\
\text { observer }\end{array}$ & $\begin{array}{c}\text { Second } \\
\text { observer }\end{array}$ & $\begin{array}{c}\text { Third } \\
\text { observer }\end{array}$ & Total \\
\hline+0.5 to +1 & 0 & 0 & 0 & 0 \\
+0.1 to +0.5 & 7.9 & 15.1 & 0 & 7.7 \\
\pm 0.1 & 49.2 & 53.2 & 20.6 & 41 \\
-0.1 to -0.5 & 41.3 & 30.1 & 78.6 & 50 \\
-0.5 to -1 & 1.6 & 1.6 & 0.8 & 1.3 \\
\hline
\end{tabular}

analyzed using repeated measures analysis of variance (ANOVA). Additionally, interobserver reliability was calculated for all magnifications using intraclass correlation coefficient (ICC).

\section{Results}

In this study, $50 \%$ of the observers underestimated the pin lengths by $0.1-0.5 \mathrm{~mm}$ compared with the actual size, whereas $7.7 \%$ of them overestimated the pin lengths by $0.1-0.5 \mathrm{~mm}$ compared with the actual size. Errors greater than $+0.5 \mathrm{~mm}$ did not occur in any of the measurements. Error ranges for pin length measurement sat all magnifications are presented in Table 1.

On the other hand, $65 \%$ of the observers overestimated the pin diameters by $0.1-0.5 \mathrm{~mm}$ compared with the actual size, whereas $1.6 \%$ of them underestimated the pin diameters by $0.1-0.5 \mathrm{~mm}$ compared with the actual size. Errors greater than $+5.0 \mathrm{~mm}$ or smaller than $-5.0 \mathrm{~mm}$ did not occur in any of the measurements. Error ranges for pin diameter measurements at all magnifications are shown in Table 2.

The mean absolute values of differences between the radiographic measurements of lengths and the actual lengths of the pins at $100 \%, 200 \%$ and $400 \%$ magnifications were 0.2206 , 0.2063 , and $0.1984 \mathrm{~mm}$, respectively. According to the results of repeated measures ANOVA, there was no significant difference in the accuracy of pin length measurements at different magnifications $(P>0.05)$. Minimum, maximum, mean and standard deviation of differences of pin length measurements at all magnifications are presented in Table 3.

\begin{tabular}{lcccc}
\hline \multicolumn{5}{c}{ Table 2. Error ranges of pin diameter measurements at all } \\
magnifications by the three observers \\
\hline Error range & $\begin{array}{c}\text { First } \\
\text { observer }\end{array}$ & $\begin{array}{c}\text { Second } \\
\text { observer }\end{array}$ & $\begin{array}{c}\text { Third } \\
\text { observer }\end{array}$ & Total \\
\hline+0.5 to +1 & 0 & 0 & 0 & 0 \\
+0.1 to +0.5 & 55.6 & 57.9 & 81.7 & 65 \\
\pm 0.1 & 39.7 & 42.1 & 18.3 & 33.4 \\
-0.1 to -0.5 & 4.7 & 0 & 0 & 1.6 \\
-0.5 to -1 & 0 & 0 & 0 & 0 \\
\hline
\end{tabular}

Table 3. Minimum, maximum, mean and SD of pin length measurement differences at all magnifications

\begin{tabular}{ccccc}
\hline Magnification & $\begin{array}{c}\text { Minimum } \\
\text { difference }\end{array}$ & $\begin{array}{c}\text { Maximum } \\
\text { difference }\end{array}$ & Mean & SD \\
\hline 100 & 0.00 & 0.60 & 0.2206 & 0.14986 \\
200 & 0.00 & 0.50 & 0.2063 & 0.12583 \\
400 & 0.00 & 0.50 & 0.1984 & 0.12266 \\
\hline
\end{tabular}

\section{Table 4. The ICC results forth measurement of pin lengths and pin diameters at different magnifications}

\begin{tabular}{llll}
\hline \multicolumn{1}{c}{ Magnification } & & & \\
Variable & $\mathbf{1 0 0 \%}$ & $\mathbf{2 0 0} \%$ & $\mathbf{4 0 0 \%}$ \\
\hline Length & 0.285 & 0.707 & 0.479 \\
Diameter & 0.078 & 0.469 & 0.587 \\
\hline
\end{tabular}

The mean absolute values of differences between the radiographic measurements of pin diameters and actual diameters of the pins were $0.1960,0.2143$, and $0.2047 \mathrm{~mm}$ at $100 \%$, $200 \%$, and $400 \%$ magnifications, respectively. According to the results of repeated measures ANOVA, there was no significant difference in the accuracy of pin diameter measurements using different magnifications $(P>0.05)$. To calculate interobserver reliability for pin length measurements, we used the ICC test. At all magnifications, interobserver reliability was estimated to be $<0.6$. For measurement of pin diameters, interobserver agreement was 0.7 at $200 \%$ magnification and it was $<0.6$ at other magnifications. The ICC test results regarding the measurement of pin lengths and diameters at all magnifications are presented in Table 4.

\section{Discussion}

The CBCT enhancement software programs facilitate and improve the diagnostic accuracy of CBCT measurements for oral and maxillofacial radiologists. Three-dimensional image reconstruction in different planes and enabling linear and angular measurements are some of the features of $\mathrm{CBCT} .^{20}$

This study aimed to evaluate the accuracy of CBCT linear measurements using New Tom VGi device at different image magnifications. According to the obtained results, the magnification level had no effect on the accuracy of linear measurements. Reliability of pin diameter measurements at all magnifications was below the acceptable range, whereas it was good at $200 \%$ magnification for pin length measurements. 
The low interobserver reliability was not clinically significant due to low variation among the measurements and the acceptable accuracy of measurements at all magnifications.

According to the findings of Ganguly et al., CBCT imaging is required to evaluate the quality and quantity of the available bone for implant placement, and to determine the exact location of anatomical structures. They also concluded that $<1 \mathrm{~mm}$ error in radiographic measurements for implant placement was clinically acceptable. ${ }^{21}$ Accordingly, errors in all measurements in our study were within the clinically acceptable range.

In the current study, pin lengths were mostly underestimated by $0.1-0.5 \mathrm{~mm}$ compared with the actual size, while pin diameters were mostly overestimated by $0.1-0.5 \mathrm{~mm}$ compared with the actual size. In a study conducted by Fatemitabar et $\mathrm{al}^{22}$, the accuracy of linear measurements made by CBCT (Planmeca) was compared with that of CT 64-channel, and the mean difference of measurements from the actual size was estimated to be +0.37 to $+0.58 \mathrm{~mm}$ using $\mathrm{CBCT}$, and +0.37 to $+0.72 \mathrm{~mm}$ using CT.

Image quality and accuracy of linear measurements made by CBCT are affected by different factors, such as the material of the pin (in pin length measurements), thickness of sections, reconstruction angle and device rotation during imaging. ${ }^{12,16}$

The findings of the current study indicated that magnification level had no effect on the accuracy of linear measurements. Moreover, it was observed that reliability of pin diameter measurements at all magnifications was below the acceptable range, whereas it was good at $200 \%$ magnification for pin length measurements. Low rate of inter observer agreement was probably due to low variation among the measurements. Sherrard et al., ${ }^{12}$ and Moshfeghi et al. ${ }^{13}$ reported that size of voxels had no significant effect on the accuracy of CBCT linear measurements or interobserver agreement.

Although the aforementioned studies evaluated the effects of factors other than magnification on the accuracy of linear measurements, similar to our findings, they reported no significant association between the studied parameters and the accuracy of linear measurements. However, the interobserver agreement in the above-mentioned studies was much higher than the value in the current study; the ICC was reported to be $>0.99$ in both of the aforementioned studies. Lower interobserver agreement in our study was due to the low variation among the measurements.

In the current study, there was no significant difference in the accuracy of linear measurements made at different image magnifications. In a study conducted by Hashem et al., there was no significant difference between the radiographic measurements obtained at two different device rotations and direct measurements. Similar to our study, Hashem et al. ${ }^{14}$ reported no significant correlation between the accuracy of CBCT linear measurements and the studied variable; however, inter observer and intraobserver agreements were reported to be good.

According to the results obtained by Nikneshan et al., changing the reconstruction angle from $-12^{\circ}$ to $+12^{\circ}$ decreased the accuracy of CBCT linear measurements. They reported that error rate was below $0.5 \mathrm{~mm}$ at all reconstruction angles, which was clinically acceptable. ${ }^{15}$ Similar to our study, titanium pins were inserted into sheep mandibles in the study conducted by Nikneshan et al. ${ }^{15}$ It is noteworthy that titanium pins were used due to low atomic number of the metal, ${ }^{16}$ which, in turn, decreases the metal artifacts in CT. Another similarity between their study and ours was that in both of the studies the mandibles were immersed in water to simulate soft tissues. However, unlike our study, researchers only measured the length of pins for evaluating the accuracy of measurements in the study conducted by Nikneshan et al. ${ }^{15}$

To date, no study has evaluated the effect of image magnification on the accuracy of linear measurements. However, several studies have investigated the effects of image magnification on radiographic diagnostic accuracy.

According to the results of the current study, image magnification has no effect on the accuracy of linear measurements. In one research, Kositbowornchai et al. reported similar results regarding the diagnostic accuracy of root fractures at different image magnifications (50\%, 100\% and 200\%). Consistent with our findings, they observed no significant difference in the criteria for diagnostic accuracy namely positive and negative predictive values, sensitivity and specificity among three different magnifications. ${ }^{17}$ Effects of magnification on interobserver reliability were not assessed in the aforementioned study.

In another study, Kositbowornchai et al. evaluated the effects of changes in sharpness, magnification and pseudo color on the diagnostic accuracy of digital two-dimensional radiographs for occlusal caries. According to their results, there was no significant relationship between these variables and the diagnostic accuracy of radiography, which is consistent with our findings.

The rate of interobserver agreement in the study by Kositbowornchai et al. ${ }^{17,18}$ was reported to be good according to Cohen's kappa coefficient. Therefore, the rate of this parameter was higher in the study by Kositbowornchai et al. compared with the rate in our study.

In another study, Morais et al. evaluated the effect of image magnification $(100 \%, 200 \%$, and $400 \%)$ in two-dimensional digital radiography on the accuracy of diagnosis of periodontal bone lesions in vitro. Similar to our study, there was no significant difference in the diagnostic accuracy at different image magnifications. ${ }^{19}$ In addition, rate of intraobserver agreement for each observer was reported to be moderate at $100 \%, 200 \%$ and $400 \%$ magnifications.

\section{Conclusion}

In conclusion, it could be stated that the accuracy of linear measurements made on CBCT images is not affected by image magnification. Moreover, interobserver reliability of the measurement of pin diameter was good at $200 \%$, while it was below the acceptable range at other magnifications. Interobserver reliability was below the acceptable range for the measurement of pin lengths at all magnifications. The low interobserver reliability was not of clinical significance because of low variation among the measurements and acceptable accuracy of measurements at all magnifications.

\section{Conflicts of Interest}

None. 


\section{References}

1. Benavides E, Rios HF, Ganz SD, An CH, Resnik R, Reardon GT, et al. Use of cone beam computed tomography in implant dentistry: the International Congress of Oral Implantologists consensus report. Implant Dent. 2012;21:78-86.

2. Al-Ekrish AA, Ekram M. A comparative study of the accuracy and reliability of multidetector computed tomography and cone beam computed tomography in the assessment of dental implant site dimensions. Dentomaxillofac Radiol. 2011;40:67-75.

3. Sheikhi M, Ghorbanizadeh S, Abdinian M, Goroohi H, Badrian H. Accuracy of linear measurements of galileos cone beam computed tomography in normal and different head positions. Int J Dent. 2012;2012:214954.

4. Ziegler CM, Woertche R, Brief J, Hassfeld S. Clinical indications for digital volume tomography in oral and maxillofacial surgery. Dentomaxillofac Radiol. 2002;31:126-130.

5. Guerrero ME, Jacobs R, Loubele M, Schutyser F, Suetens P, van Steenberghe D. State-of-the-art on cone beam CT imaging for preoperative planning of implant placement. Clin Oral Investig. 2006;10:1-7.

6. Hellak A, Schmidt N, Schauseil M, Stein S, Drechsler T, KorbmacherSteiner HM. Influence of Invisalign treatment with interproximal enamel reduction (IER) on bone volume for adult crowding: a retrospective threedimensional cone beam computed tomography study. BMC Oral Health 2016;16:83.

7. Yamamoto K, Ueno K, Seo K, Shinohara D. Development of dentomaxillofacial cone beam X-ray computed tomography system. Orthod Craniofac Res. 2003;6:160-162.

8. Yepes JF, Al-Sabbagh M. Use of cone-beam computed tomography in early detection of implant failure. Dent Clin North Am. 2015:59:41-56.

9. Cavalcanti MG, Rocha SS, Vannier MW. Craniofacial measurements based on 3D-CT volume rendering: implications for clinical applications. Dentomaxillofac Radiol. 2004;33:170-176.

10. John GP, Joy TE, Mathew J, Kumar VR. Fundamentals of cone beam computed tomography for a prosthodontist. J Indian Prosthodont Soc. 2015;15:8-13.

11. Tyndall DA, Rathore S. Cone-beam CT diagnostic applications: caries, periodontal bone assessment, and endodontic applications. Dent Clin North Am. 2008;52:825-841, vii.
12. Sherrard JF, Rossouw PE, Benson BW, Carrillo R, Buschang PH. Accuracy and reliability of tooth and root lengths measured on cone-beam computed tomographs. Am J Orthod Dentofacial Orthop. 2010;137:S100-S108.

13. Moshfeghi M, Tavakoli MA, Hosseini ET, Hosseini AT, Hosseini IT. Analysis of linear measurement accuracy obtained by cone beam computed tomography (CBCT-NewTom VG). Dent Res J (Isfahan). 2012;9:S57-S62.

14. Hashem D, Brown JE, Patel S, Mannocci F, Donaldson AN, Watson TF, et al. An in vitro comparison of the accuracy of measurements obtained from high- and low-resolution cone-beam computed tomography scans. J Endod. 2013;39:394-397.

15. Nikneshan S, Aval SH, Bakhshalian S, Shahab S, Mohammadpour M, Sarikhani S. Accuracy of linear measurement using cone-beam computed tomography at different reconstruction angles. Imaging Sci Dent. 2014;44:257-262.

16. Boas FE, Fleischmann D. Evaluation of two iterative techniques for reducing metal artifacts in computed tomography. Radiology. 2011;259:894-902.

17. Kositbowornchai S, Sikram S, Nuansakul R, Thinkhamrop B. Root fracture detection on digital images: effect of the zoom function. Dent Traumatol. 2003;19:154-159.

18. Kositbowornchai S, Basiw M, Promwang Y, Moragorn H, Sooksuntisakoonchai N. Accuracy of diagnosing occlusal caries using enhanced digital images. Dentomaxillofac Radiol. 2004;33:236-240.

19. de Morais J, Sakakura CE, Lofferdo LC, Scaf G. Accuracy of zoomed digital image in the detection of periodontal bone defect: in vitro study. Dentomaxillofac Radiol. 2006;35:139-142

20. Rangel FA, Maal TJ, Bronkhorst EM, Breuning KH, Schols JG, Bergé SJ, et al. Accuracy and reliability of a novel method for fusion of digital dental casts and cone beam computed tomography scans. PloS One. 2013;8:e59130.

21. Ganguly R, Ruprecht A, Vincent S, Hellstein J, Timmons S, Qian F. Accuracy of linear measurement in the Galileos cone beam computed tomography under simulated clinical conditions. Dentomaxillofac Radiol. 2011;40:299-305.

22. Fatemitabar SA, Nikgoo A. Multichannel computed tomography versus cone-beam computed tomography: linear accuracy of in vitro measurements of the maxilla for implant placement. Int J Oral Maxillofacial Implants. 2010;25:499-505. 